\title{
Impact and Estimation of Balance Coordinate System Rotations and Translations in Wind-Tunnel Testing
}

\author{
Kenneth G. Toro* and Peter A. Parker ${ }^{\dagger}$ \\ NASA Langley Research Center, Hampton, Virginia, 23681, USA
}

\begin{abstract}
Discrepancies between the model and balance coordinate systems lead to biases in the aerodynamic measurements during wind-tunnel testing. The reference coordinate system relative to the calibration coordinate system at which the forces and moments are resolved is crucial to the overall accuracy of force measurements. This paper discuses sources of discrepancies and estimates of coordinate system rotation and translation due to machining and assembly differences. A methodology for numerically estimating the coordinate system biases will be discussed and developed. Two case studies are presented using this methodology to estimate the model alignment. Examples span from angle measurement system shifts on the calibration system to discrepancies in actual wind-tunnel data. The results from these case-studies will help aerodynamic researchers and force balance engineers to better the understand and identify potential differences in calibration systems due to coordinate system rotation and translation.
\end{abstract}

\section{Nomenclature}

$\beta \quad$ Regression Coefficients

$\boldsymbol{F} \quad$ Force Vector, lbs.

$\boldsymbol{M}$ Moment Vector, in-lbs.

$\phi \quad$ Roll Angle, Deg.

$\psi \quad$ Yaw Angle, Deg.

$\theta \quad$ Pitch Angle, Deg.

$\tilde{a} \quad$ Skew-Symmetric Matrix

$T \quad$ Rotation Transformation Matrix

y $\quad$ FMS Voltages

LaRC Langley Research Center

NASA National Aeronautics and Space Administration

\section{Introduction}

Force measurement systems (FMS) rely on high-precision fits and quality-assurance measurements to define the reference coordinate system that impacts transfer and use of the instrument. Any offset in position or rotation between the balance and the model coordinate systems will manifest in measurement discrepancies. A change in attachment to the metric, or sensing, end of the balance will result in these discrepancies, since each attachment will yield slightly different alignments. Resulting discrepancies may not be detectable with a single wind-tunnel dataset due to the lack of precise check loads. But, these discrepancies may become apparent when results for a wind-tunnel test are compared to separate tests, whether it is a previous entry or an independent run at another facility using the same model.

For the reasons stated above, angle measurement is an essential part a FMS. During the calibration of a FMS, a coordinate system is affixed by the angle reference that is used to define balance forces during the

\footnotetext{
* Research Engineer, System Engineering and Engineering Methods, NASA Langley Research Center

†Team Lead, Advanced Measurement and Data Systems, NASA Langley Research Center
} 
calibration. For most calibration systems this fixing of the coordinate system is due to having the applied load referenced to gravity. Typically, the calibration body used will define the coordinate system of the FMS and it will incorporate any misalignments that may exist between the calibration body and the balance.

This paper discusses the impart of balance coordinate systems and their rotations. The development of the framework for estimating the rotations and translations that may exist due to the calibration, model hardware, and angle measurement systems. Numerical routines will be implemented to estimate the required rotations and translations to identify if a set of rotations and translation can bring two independent sets of force measurements into agreement.

\section{Methodology}

Typically, the coordinate systems used for an FMS and wind-tunnel testing do not adhere to the conventional right-hand-rule system. Instead, the positive direction for normal and axial force are opposite of their respective $z$ and $x$ axes, as shown in Figure $1 .^{1}$ This was historically done due to the lift and drag primarily acting in these directions for an upright aircraft model. This coordinate system will be used throughout this paper and for any of the following derivations. It is important to note that the moments do behave in the same manner as for a right-hand coordinate system.

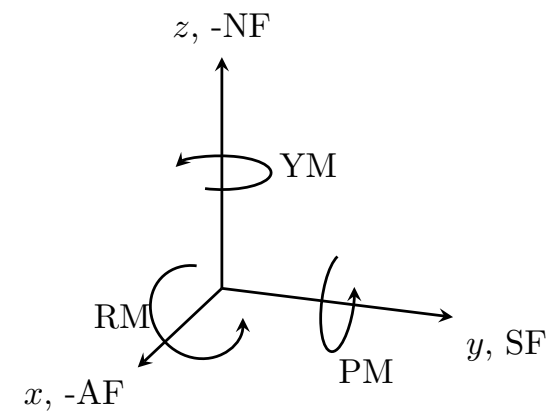

Figure 1. Balance Coordinate System

\section{A. Coordinate System Rotation and Translation}

Angular rotations of $\phi, \theta$, and $\psi$ can be applied to the balance coordinate system about the $x, y$, and $z$ axes, respectively. These rotations on the coordinate system are illustrated in Figure 2, where $x^{\prime}$ and $x^{\prime \prime}$ are steps between an initial position of $x_{I}$ and a final position of $x_{F}$. Note that this rotation convention rotates the system in $z-y-x$ order and is used in the following formulations.

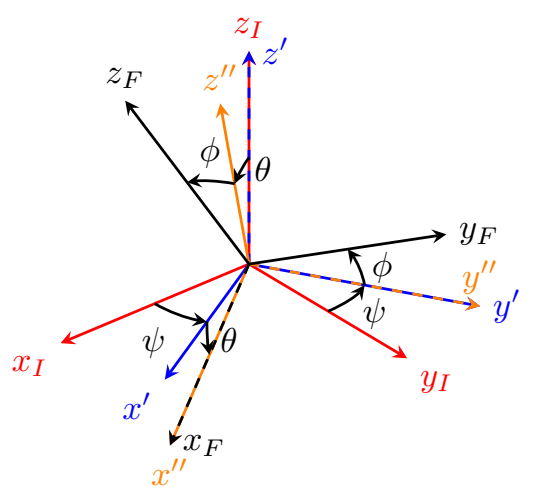

Figure 2. Coordinate System Rotation

These rotations represent the delta misalignments that exist between two coordinate systems, and are used to derive rotational transformations between an initial and final coordinate systems. A vector $\boldsymbol{v}$ can be 
represented in a new rotated coordinate system by $\boldsymbol{v}^{\prime},{ }^{2,3}$

$$
\boldsymbol{v}^{\prime}=\left[\begin{array}{ccc}
C_{\psi} C_{\theta} & S_{\psi} C_{\theta} & -S_{\theta} \\
S_{\psi} S_{\theta} S_{\phi}+S_{\psi} C_{\phi} & S_{\psi} S_{\theta} S_{\phi}+C_{\psi} C_{\phi} & C_{\theta} S_{\phi} \\
C_{\psi} S_{\theta} C_{\phi}+S_{\psi} S_{\phi} & S_{\psi} S_{\theta} C_{\phi}-C_{\psi} S_{\phi} & C_{\theta} C_{\phi}
\end{array}\right] \boldsymbol{v}=T_{(\phi, \theta, \psi)} \boldsymbol{v}
$$

where $C_{\theta}$ and $S_{\theta}$ are the cosine and sine functions applied to the $\theta$ angle. Equation 1 is used to rotate the force vector between the two coordinate systems,

$$
\boldsymbol{F}^{\prime}=T_{(\phi, \theta, \psi)} \boldsymbol{F}=T_{(\phi, \theta, \psi)}\left[\begin{array}{c}
F_{x} \\
F_{y} \\
F_{z}
\end{array}\right]=T_{(\phi, \theta, \psi)}\left[\begin{array}{c}
-\mathrm{AF} \\
\mathrm{SF} \\
-\mathrm{NF}
\end{array}\right]
$$

where $\boldsymbol{F}$ is the applied force vector with the components $F_{i}$ that represents the force along the $i^{\text {th }}$ axis. Note that the translation from axis based forces to the balance definition in Figure 1 is shown by the last term in Equation 2.

Moments, $\boldsymbol{M}$, undergo the same rotation transformation, but require an additional step for any translations in the coordinate system. The translation of a coordinate system defined by a displacement vector $\boldsymbol{r}$ represents the movement of the coordinate system to a new location. Moments at the new reference location and in a rotated coordinate system can be found by,

$$
\boldsymbol{M}^{\prime}=T_{(\phi, \theta, \psi)}(\boldsymbol{M}-(\boldsymbol{r} \times \boldsymbol{F}))
$$

where $\boldsymbol{M}^{\prime}$ are moments with respect to the translated coordinate system, $\boldsymbol{M}$ are moments with respect to the original coordinate system, and $v=\left[\begin{array}{lll}x & y & z\end{array}\right]^{T}$. The cross product in Equation 3 can be replaced with the skew-symmetric matrix, $\tilde{a}$, to convert the equation over to matrix notation,

$$
\boldsymbol{M}^{\prime}=T_{(\phi, \theta, \psi)}(\boldsymbol{M}-\tilde{a} \boldsymbol{F})=T_{(\phi, \theta, \psi)}\left(\boldsymbol{M}-\left[\begin{array}{ccc}
0 & -z & y \\
z & 0 & x \\
-y & x & 0
\end{array}\right] \boldsymbol{F}\right)
$$

\section{B. Transformation Estimation}

With the framework to rotate and translate the forces and moments to another coordinate system, the angles and offsets between two systems can be estimated given two sets of loads. Let the forces and moments of system 1 be noted by $F_{1}$ and $M_{1}$, and similarly $F_{2}$ and $M_{2}$ for system 2 . For these pairs of forces and moments, there exists a $\hat{T}$ and $\hat{\tilde{a}}$ that satisfies,

$$
\begin{aligned}
\boldsymbol{F}_{2} & =\hat{T}_{(\phi, \theta, \psi)} \boldsymbol{F}_{1} \\
\boldsymbol{M}_{2} & =\hat{T}_{(\phi, \theta, \psi)}\left(\boldsymbol{M}_{1}-\hat{\tilde{a}}_{(x, y, z)} \boldsymbol{F}_{1}\right)
\end{aligned}
$$

where the hat, or caret, are used to express an estimated term, i.e. $\hat{T}$ is an estimate of $T$. Both sides of Equations 5 and 6 can be subtracted by the right hand side,

$$
\begin{aligned}
& H_{1}=\boldsymbol{F}_{2}-\boldsymbol{F}_{1}^{\prime}=\boldsymbol{F}_{2}-\hat{T}_{(\phi, \theta, \psi)} \boldsymbol{F}_{1} \\
& H_{2}=\boldsymbol{M}_{2}-\boldsymbol{M}_{1}^{\prime}=\boldsymbol{M}_{2}-\hat{T}_{(\phi, \theta, \psi)}\left(\boldsymbol{M}_{1}-\hat{\tilde{a}}_{(x, y, z)} \boldsymbol{F}_{1}\right)
\end{aligned}
$$

where both $H_{1}$ and $H_{2}$ equal zero when a set of angles and translations are found that transfer the first coordinate system to the second. With this methodology, any two sets of loads cannot be used to estimate coordinate system translations and rotations, only load sets that have a mutual relationship are applicable. An example would be comparing estimated loads that are generated using two calibration matrices but a single set of measured voltages. Methods implemented to estimated loads, $\hat{x}$, vary between facilities and 
organizations, and can be represented by a function of the measured voltages, $y_{j}$, and the calibration matrix, $M_{i}$,

$$
\hat{x}_{M i_{j}}=f\left(y_{j}, M_{i}\right)
$$

where $i$ and $j$ identifies which system's ( 1 or 2 ) data is used. For example, $\hat{x}_{M 2_{1}}$ corresponds to estimated loads using voltages from system 1 and the calibration matrix of system 2 . With two systems this can be expanded out to four different voltages/matrix combinations, which should be paired by system voltage, i.e. paring estimated loads generated by $y_{1}$ or $y_{2}$. Estimated forces and moments from $\hat{x}_{M 1_{1}}$ and $\hat{x}_{M 2_{1}}$ are substituted into Equations 7 and 8, which will allow for the solving of coordinate system rotations and translations. The system of equations can be solved using non-linear least squares, or other solvers. In this study the Levenberg-Marquardt algorithm is implemented in the solver for all cases.

\section{Significance Testing}

The methodology derived for finding a set of rotations and translations that match loads between two calibrations may find a solution despite not being significant. Statistical tools are implemented to provide an indication of whether a rotation matrix is significant given a set of load data. Misalignments resulting from the installation of a balance into a wind-tunnel model or calibration fixture one would expect small angles and displacements due to the precision machined surfaces, thus small angle approximation can be implemented on Equation 1. By assuming that $\sin (x)=x$ and $\cos (x)=1$, Equation 1 can be simplified to,

$$
T_{(\phi, \theta, \psi)}=\left[\begin{array}{ccc}
1 & \psi & -\theta \\
\psi \theta \phi+\psi & \psi \theta \phi+1 & \phi \\
\theta+\psi \phi & \psi \theta-\phi & 1
\end{array}\right]
$$

Substituting Equation 10 into Equation 2 and subtracting by $\boldsymbol{F}$ gives a representation of the difference between two coordinate systems,

$$
\Delta \boldsymbol{F}=\boldsymbol{F}^{\prime}-\boldsymbol{F}=\left[\begin{array}{ccccc}
0 F_{x} & + & \psi F_{y} & + & -\theta F_{z} \\
(\psi \theta \phi+\psi) F_{x} & + & \psi \theta \phi F_{y} & + & \phi F_{z} \\
(\theta+\psi \phi) F_{x} & + & (\psi \theta-\phi) F_{y} & + & 0 F_{z}
\end{array}\right]
$$

This shows that for small angles a linear model can be used to represent the rotation function required to equate residual loads, $\Delta F$, and one of the two loads, $F$. With the assumption that a linear relationship exists for small angles, a linear least squares regression can be utilized to estimate the following mathematical model between residuals and estimated loads,

$$
\Delta F_{i}=\hat{\beta}_{x i} F_{x}+\hat{\beta}_{y i} F_{y}+\hat{\beta}_{z i} F_{z}
$$

where the $\hat{\beta}_{j i}$ terms are the estimated coefficients, and the $i$ subscript is for the $\mathrm{i}^{\text {th }}$ component. Analysis of variance, or ANOVA, is performed to determine if the fitted linear model is statistically significant or couldn't be supported due to the presence of noise. It is suggested that the least squares and ANOVA analysis is conducted prior to implementing the non-linear optimization process to ensure that a relationship can be statistically supported.

If a linear model is found to be significant, the estimated values in the model may not relate to unique angles in Equation 1. The linear model found may include other effects than the angular change that is under investigation, such as primary sensitivity shifts, and differences in the second order terms. To evaluate the statistical significance of a set of angles found using the root-finding method discussed earlier, changes in variances of the residual errors can be tested for significant improvements. Two sets of residual errors are defined as,

$$
\begin{aligned}
\Delta F & =F_{2}-F_{1} \\
\Delta F_{T} & =F_{2}-F_{1}^{\prime}
\end{aligned}
$$

where one set is the difference between unprocessed loads, and the other is between loads from system two and rotated results from system 1 . With this format, the differences between residuals is only a results of 
implementing the angular rotations that were estimated. F-Test for variance equality is implemented to detect significant changes between the two residual variances, ${ }^{4}$

$$
F=\frac{s_{1}^{2}}{s_{2}^{2}}=\frac{s_{\Delta F_{T}}^{2}}{s_{\Delta F}^{2}}
$$

where $s_{\Delta F_{T}}^{2}$ and $s_{\Delta F}^{2}$ are sample variances of $\Delta F_{T}$ and $\Delta F$ respectively. The hypothesis under investigation is whether the angular rotation decreases the variance of the residuals,

$$
\begin{array}{ll}
H_{0}: & s_{\Delta F_{T}}^{2}=s_{\Delta F}^{2} \\
H_{1}: & s_{\Delta F_{T}}^{2}<s_{\Delta F}^{2}
\end{array}
$$

where the rejection of the null hypothesis, $H_{0}$, will signify that there is an improvement in the residual error. This improvement in residuals will signify that the rotations and translations found are significant and can be accepted to be implemented to relate the two loads.

\section{Application}

Two case studies are conducted to test the use of the developed methodology, which one is a simulated calibration and the other actual calibration data. For each of these case studies the inputs are a set of calibration matrices with a fixed set of loads. Loads created using a central composite design (CCD) based on the full-scales calibration loads. A full central composite design of 64 runs was built with factorial points set to $1 / 2^{6 / 4}$ of the calibration full scale loads. These developed loads are passed through the first calibration matrix to generate simulated voltages by reversing Equation 9. These simulated voltages then are used to solve for the loads of system 2 given the second calibration matrix. This removes any variability and outliers that may exist in the calibration data or voltages, and only compares differences that exist between the two calibration matrices.

\section{A. Case Study 1 - Simulated SVS}

The NASA LaRC Single-Vector System (SVS) is a calibration system that relies on an Angle Measurement System (AMS) that comprises of a 3-axis accelerometer, and a precision load template. ${ }^{5}$ The balance coordinate system that is defined in a SVS calibration is set by the AMS, where any AMS misalignment will manifest in the calibration matrix. In this case study, the estimated gravity vector based on AMS outputs is rotated by a constant amount to simulate a physical AMS rotation. Load templates used in the SVS calibration provides definition of the balance moment center (BMC) since it is used to define the distances to load positions. Measurement or positioning error of this load template will lead to movement of the BMC. Several cases will be demonstrated in this approach by perturbing the AMS offset angles and load template positions, as shown in Table 1 . The results of this case study demonstrate the ability of this method to estimate the offsets and show the importance of the coordinate system transfer between calibration and wind-tunnel testing and/or calibration system to calibration system.

Table 1. AMS Rotation Angles

\begin{tabular}{ccccccc}
\hline \hline Case & $\phi$ & $\theta$ & $\psi$ & $x$ & $y$ & $z$ \\
\hline 1 & 0.00 & 0.00 & 0.00 & 0.00 & 0.00 & 0.00 \\
2 & 0.02 & 0.00 & 0.00 & 0.00 & 0.00 & 0.00 \\
3 & 0.00 & 0.02 & 0.00 & 0.00 & 0.00 & 0.00 \\
4 & 0.02 & 0.02 & 0.00 & 0.00 & 0.00 & 0.00 \\
5 & -0.02 & -0.02 & 0.00 & 0.00 & 0.00 & 0.00 \\
6 & 0.00 & 0.00 & 0.00 & 0.05 & 0.00 & 0.00 \\
7 & 0.00 & 0.00 & 0.00 & 0.01 & -0.01 & 0.00 \\
8 & 0.02 & 0.02 & 0.00 & 0.01 & -0.01 & 0.00 \\
\hline \hline
\end{tabular}


The original data that is used in this case study is from a SVS calibration of the $843 \mathrm{~A}$ balance that was originally conducted in August of 2009. The full scale loads used for the balance calibration are shown in Table 2, and will be used for building the applied loads to be analyzed. Data from the calibration experiment were processed using custom scripts design to process SVS calibration data with the modifications to apply Equation 1 to all gravity vector estimates and adjusting load positions. This rotation and position offset will adjust the applied load estimates that are used to build the calibration matrix for each of the cases in this study.

Table 2. 843A Balance Full Scale Loads

\begin{tabular}{cccccc}
\hline \hline NF & AF & PM & RM & YM & SF \\
lbs. & lbs. & in-lbs. & in-lbs. & in-lbs. & lbs. \\
\hline 400 & 100 & 1250 & 300 & 1250 & 400 \\
\hline \hline
\end{tabular}

The methodology developed is designed to compare calibrations, pairings of the cases are compared. Estimates for the deltas in rotation and position between several pairs of cases are shown in Table 3. As seen in the table, results match what is expected from the case pairs, where the delta rotation and position values are the angles and offsets of Matrix 2 subtracted by those of Matrix 1.

Table 3. Simulated SVS - Delta Rotation and Position Estimates

\begin{tabular}{cccccccc}
\hline \hline Matrix 1 & Matrix 2 & $\Delta \phi$ & $\Delta \theta$ & $\Delta \psi$ & $\Delta x$ & $\Delta y$ & $\Delta z$ \\
\hline 1 & 2 & 0.020 & -0.000 & 0.000 & -0.000 & 0.000 & 0.000 \\
1 & 3 & -0.000 & 0.020 & 0.000 & -0.000 & 0.000 & 0.000 \\
1 & 4 & 0.020 & 0.020 & 0.000 & -0.000 & 0.000 & 0.000 \\
1 & 5 & -0.020 & -0.020 & -0.000 & 0.000 & -0.000 & -0.000 \\
2 & 3 & -0.020 & 0.020 & -0.000 & -0.000 & -0.000 & 0.000 \\
2 & 4 & -0.000 & 0.020 & -0.000 & -0.000 & 0.000 & 0.000 \\
2 & 5 & -0.040 & -0.020 & 0.000 & 0.000 & -0.000 & -0.000 \\
3 & 4 & 0.020 & -0.000 & 0.000 & -0.000 & 0.000 & 0.000 \\
3 & 5 & -0.020 & -0.040 & -0.000 & 0.000 & -0.000 & -0.000 \\
4 & 5 & -0.040 & -0.040 & 0.000 & 0.000 & -0.000 & -0.000 \\
4 & 3 & -0.020 & 0.000 & -0.000 & 0.000 & -0.000 & -0.000 \\
4 & 5 & -0.040 & -0.040 & 0.000 & 0.000 & -0.000 & -0.000 \\
1 & 6 & 0.000 & -0.000 & -0.000 & 0.050 & 0.000 & -0.000 \\
1 & 7 & -0.000 & -0.000 & -0.000 & 0.010 & -0.010 & -0.000 \\
1 & 8 & 0.020 & 0.020 & 0.000 & 0.010 & -0.010 & -0.000 \\
\hline \hline
\end{tabular}

Results of the hypothesis are shown in Table 4 and its associated p-values are shown Table 5 . In the table of the hypothesis results, a value of 1 indicates that the null hypothesis, $H_{0}$, is rejected based on a 0.05 level of significances. These results show that there is at least two or more components that show improved residual results with the estimated angles and offsets. It is expected that two or more components will show a significant change in residuals if a rotation or offset is required, due to the coupling between components as shown in Equations 1 and 4. From the p-value results, it is seen that values that there is a strong signal to noise ratio for the data, which is signified by small values for cases where the null hypothesis is rejected.

\section{B. Case Study 2 - NTF-113C Calibration Data}

This case study compares four calibrations of the NTF-113C force balance that were conducted on two different calibration systems. The NASA LaRC SVS and Calspan automated balance calibration system (ABCS) were used to produce two sets of calibrations. ${ }^{5,6}$ Both calibration systems have a single fixed coordinate system, where the ABCS monitors the balance coordinate system with linear displacement sensor sand the SVS was described in the previous section. Unlike these systems, manual calibration stands have 
Table 4. Simulated SVS - Component F-Test Hypothesis

\begin{tabular}{cccccccc}
\hline \hline & & \multicolumn{7}{c}{ Hypothesis Test Results } \\
Matrix 1 & Matrix 2 & NF & AF & PM & RM & YM & SF \\
\hline 1 & 2 & 1 & 0 & 1 & 1 & 1 & 1 \\
1 & 3 & 1 & 1 & 0 & 0 & 1 & 0 \\
1 & 4 & 1 & 1 & 1 & 0 & 1 & 1 \\
1 & 5 & 1 & 1 & 1 & 0 & 1 & 1 \\
2 & 3 & 1 & 1 & 1 & 0 & 1 & 1 \\
2 & 4 & 1 & 1 & 0 & 0 & 1 & 0 \\
2 & 5 & 1 & 1 & 1 & 0 & 1 & 1 \\
3 & 4 & 1 & 0 & 1 & 1 & 1 & 1 \\
3 & 5 & 1 & 1 & 1 & 0 & 1 & 1 \\
4 & 5 & 1 & 1 & 1 & 0 & 1 & 1 \\
4 & 3 & 1 & 0 & 1 & 1 & 1 & 1 \\
4 & 5 & 1 & 1 & 1 & 0 & 1 & 1 \\
1 & 6 & 0 & 0 & 1 & 0 & 1 & 0 \\
1 & 7 & 0 & 0 & 1 & 1 & 1 & 0 \\
1 & 8 & 1 & 1 & 1 & 1 & 1 & 1 \\
\hline \hline
\end{tabular}

Table 5. Simulated SVS - Component F-Test P-Values

\begin{tabular}{cccccccc}
\hline \hline & & \multicolumn{5}{c}{ Hypothesis P-Value } \\
Matrix 1 & Matrix 2 & NF & AF & PM & RM & YM & SF \\
\hline 1 & 2 & $3.6 \mathrm{e}-183$ & $5.0 \mathrm{e}-01$ & $1.6 \mathrm{e}-162$ & $3.0 \mathrm{e}-09$ & $5.0 \mathrm{e}-182$ & $1.5 \mathrm{e}-232$ \\
1 & 3 & $1.4 \mathrm{e}-200$ & $6.8 \mathrm{e}-249$ & $4.8 \mathrm{e}-01$ & $1.0 \mathrm{e}+00$ & $1.1 \mathrm{e}-156$ & $5.0 \mathrm{e}-01$ \\
1 & 4 & $8.6 \mathrm{e}-184$ & $6.1 \mathrm{e}-196$ & $2.2 \mathrm{e}-59$ & $1.0 \mathrm{e}+00$ & $1.2 \mathrm{e}-177$ & $2.6 \mathrm{e}-192$ \\
1 & 5 & $8.4 \mathrm{e}-184$ & $6.4 \mathrm{e}-196$ & $2.2 \mathrm{e}-59$ & $1.0 \mathrm{e}+00$ & $1.4 \mathrm{e}-177$ & $2.6 \mathrm{e}-192$ \\
2 & 3 & $7.7 \mathrm{e}-184$ & $8.3 \mathrm{e}-194$ & $2.3 \mathrm{e}-59$ & $1.0 \mathrm{e}+00$ & $1.5 \mathrm{e}-180$ & $3.5 \mathrm{e}-192$ \\
2 & 4 & $1.4 \mathrm{e}-200$ & $6.9 \mathrm{e}-249$ & $4.8 \mathrm{e}-01$ & $1.0 \mathrm{e}+00$ & $1.2 \mathrm{e}-156$ & $4.9 \mathrm{e}-01$ \\
2 & 5 & $2.5 \mathrm{e}-183$ & $5.7 \mathrm{e}-173$ & $7.1 \mathrm{e}-82$ & $1.0 \mathrm{e}+00$ & $2.4 \mathrm{e}-180$ & $2.1 \mathrm{e}-211$ \\
3 & 4 & $3.6 \mathrm{e}-183$ & $5.0 \mathrm{e}-01$ & $1.1 \mathrm{e}-162$ & $3.0 \mathrm{e}-09$ & $5.0 \mathrm{e}-182$ & $1.5 \mathrm{e}-232$ \\
3 & 5 & $1.9 \mathrm{e}-185$ & $5.8 \mathrm{e}-218$ & $1.1 \mathrm{e}-37$ & $1.0 \mathrm{e}+00$ & $6.4 \mathrm{e}-171$ & $1.6 \mathrm{e}-170$ \\
4 & 5 & $8.4 \mathrm{e}-184$ & $6.4 \mathrm{e}-196$ & $2.2 \mathrm{e}-59$ & $1.0 \mathrm{e}+00$ & $2.7 \mathrm{e}-177$ & $2.6 \mathrm{e}-192$ \\
4 & 3 & $3.6 \mathrm{e}-183$ & $5.0 \mathrm{e}-01$ & $1.1 \mathrm{e}-162$ & $3.0 \mathrm{e}-09$ & $4.9 \mathrm{e}-182$ & $1.5 \mathrm{e}-232$ \\
4 & 5 & $8.4 \mathrm{e}-184$ & $6.4 \mathrm{e}-196$ & $2.2 \mathrm{e}-59$ & $1.0 \mathrm{e}+00$ & $2.7 \mathrm{e}-177$ & $2.6 \mathrm{e}-192$ \\
1 & 6 & $5.0 \mathrm{e}-01$ & $5.0 \mathrm{e}-01$ & $0.0 \mathrm{e}+00$ & $5.0 \mathrm{e}-01$ & $0.0 \mathrm{e}+00$ & $5.0 \mathrm{e}-01$ \\
1 & 7 & $5.0 \mathrm{e}-01$ & $5.0 \mathrm{e}-01$ & $0.0 \mathrm{e}+00$ & $0.0 \mathrm{e}+00$ & $0.0 \mathrm{e}+00$ & $4.9 \mathrm{e}-01$ \\
1 & 8 & $8.6 \mathrm{e}-184$ & $6.0 \mathrm{e}-196$ & $2.1 \mathrm{e}-132$ & $8.9 \mathrm{e}-53$ & $5.6 \mathrm{e}-251$ & $2.6 \mathrm{e}-192$ \\
\hline \hline
\end{tabular}


no truly fixed coordinate system due to typically relying on a level that is placed on four or more separate lands. Having a fixed coordinate system during the entire calibration makes this case a good example of implementing this method, since a single rotation should be present during each of the calibrations.

Table 6. NTF-113C Calibration Full Scale Loads

\begin{tabular}{cccccc}
\hline \hline $\begin{array}{c}\text { NF } \\
\text { lbs. }\end{array}$ & $\begin{array}{c}\text { AF } \\
\text { lbs. }\end{array}$ & $\begin{array}{c}\text { PM } \\
\text { in-lbs. }\end{array}$ & $\begin{array}{c}\text { RM } \\
\text { in-lbs. }\end{array}$ & $\begin{array}{c}\text { YM } \\
\text { in-lbs. }\end{array}$ & $\begin{array}{c}\text { SF } \\
\text { lbs. }\end{array}$ \\
\hline 3000 & 200 & 5000 & 3600 & 3000 & 2000 \\
\hline \hline
\end{tabular}

The NTF-113C is a typical monolithic balance used at NASA LaRC National Transonic Facility (NTF). Load ranges used to define the input load matrix are listed in Table 6 and are not the design full-scale loads since the calibrations were done at lower ranges. Not exercising the full-scale range of the balance can impact the rotations and offsets estimation due to lower balance resolution, but is not a concern since both calibrations have similar ranges.

Results from pairing each of the 4 matrices are shown in Table 7, where the true difference are not know. From these results, the pairs of the same system, i.e. SVS 1 and SVS 2, have small estimates for rotations and offsets, which is expected from identical calibration systems. Another observation from the estimates, are the similar rotation and offset estimates for all comparisons between SVS and ABCS. This suggests that angular offsets may exist between the two systems with roll being the largest value of approximately 0.1 degrees. Note that multiple random starting locations for the solver were tested, with identical results for starts within 2 degrees and 1 inch.

Table 7. NTF-113C - Delta Rotation and Position Estimates

\begin{tabular}{cccccccc}
\hline \hline Matrix 1 & Matrix 2 & $\Delta \phi$ & $\Delta \theta$ & $\Delta \psi$ & $\Delta x$ & $\Delta y$ & $\Delta z$ \\
\hline SVS 1 & SVS 2 & 0.004 & -0.007 & 0.006 & 0.000 & 0.001 & 0.000 \\
SVS 1 & ABCS 1 & -0.097 & 0.035 & -0.010 & -0.003 & 0.006 & -0.012 \\
SVS 1 & ABCS 2 & -0.092 & 0.027 & -0.014 & -0.002 & 0.007 & -0.007 \\
SVS 2 & ABCS 1 & -0.101 & 0.042 & -0.016 & -0.003 & 0.005 & -0.012 \\
SVS 2 & ABCS 2 & -0.096 & 0.034 & -0.020 & -0.002 & 0.006 & -0.007 \\
ABCS 1 & ABCS 2 & 0.004 & -0.008 & -0.004 & 0.001 & 0.001 & 0.005 \\
\hline \hline
\end{tabular}

Hypothesis test results and p-values are shown in Tables 4 and 5 for the NTF-113C rotation and offset estimates. It can be seen that for the similar system cases, the results are found to be insignificant other than rolling moment of SVS1-SVS2. For this rolling moment result, it can be deemed incorrect based on the results from Table 7, due to the near zero estimates and the p-value not much smaller than 0.05. Another anomaly is the possible failure to reject the null hypothesis for normal force in the SVS 2 vs. ABCS 1 case. From the angular offset estimates, it would be expected that a roll angle of -0.101 would yield a large enough change in normal force and side force based on Equation 1. This near miss is also seen in the p-value results again, since the value of 0.058 is not much larger than 0.05 .

Table 8. NTF-113C - Component F-Test Hypothesis

\begin{tabular}{cccccccc}
\hline \hline & & \multicolumn{7}{c}{ Hypothesis Test Results } \\
Matrix 1 & Matrix 2 & NF & AF & PM & RM & YM & SF \\
\hline SVS 1 & SVS 2 & 0 & 0 & 0 & 1 & 0 & 0 \\
SVS 1 & ABCS 1 & 1 & 1 & 1 & 1 & 0 & 1 \\
SVS 1 & ABCS 2 & 1 & 1 & 1 & 0 & 0 & 1 \\
SVS 2 & ABCS 1 & 0 & 1 & 1 & 1 & 0 & 1 \\
SVS 2 & ABCS 2 & 1 & 1 & 1 & 0 & 0 & 1 \\
ABCS 1 & ABCS 2 & 0 & 0 & 0 & 0 & 0 & 0 \\
\hline \hline
\end{tabular}

From theory, the hypothesis tests should be able to determine the significance of the offset estimates, 
since the moments are dependent on load locations. Offset estimates were found to be small and several of hypothesis results were expected to show insignificances. The way that the significance testing was implemented for the moments allowed for dependence on the angles, thus improvements in the moment variances can be a result of both rotations and offsets.

Table 9. NTF113C - Component F-Test P-Values

\begin{tabular}{cccccccc}
\hline \hline & & \multicolumn{7}{c}{ Hypothesis P-Value } \\
Matrix 1 & Matrix 2 & NF & AF & PM & RM & YM & SF \\
\hline SVS 1 & SVS 2 & $5.2 \mathrm{e}-01$ & $2.1 \mathrm{e}-01$ & $7.2 \mathrm{e}-01$ & $4.2 \mathrm{e}-02$ & $4.2 \mathrm{e}-01$ & $4.1 \mathrm{e}-01$ \\
SVS 1 & ABCS 1 & $3.9 \mathrm{e}-02$ & $5.7 \mathrm{e}-13$ & $4.4 \mathrm{e}-03$ & $8.9 \mathrm{e}-06$ & $4.0 \mathrm{e}-01$ & $1.5 \mathrm{e}-04$ \\
SVS 1 & ABCS 2 & $4.0 \mathrm{e}-04$ & $4.5 \mathrm{e}-06$ & $1.2 \mathrm{e}-02$ & $6.8 \mathrm{e}-02$ & $3.2 \mathrm{e}-01$ & $6.2 \mathrm{e}-07$ \\
SVS 2 & ABCS 1 & $5.8 \mathrm{e}-02$ & $2.1 \mathrm{e}-34$ & $6.0 \mathrm{e}-03$ & $2.4 \mathrm{e}-05$ & $3.3 \mathrm{e}-01$ & $1.2 \mathrm{e}-06$ \\
SVS 2 & ABCS 2 & $5.8 \mathrm{e}-04$ & $4.2 \mathrm{e}-12$ & $1.2 \mathrm{e}-02$ & $9.2 \mathrm{e}-02$ & $2.9 \mathrm{e}-01$ & $3.6 \mathrm{e}-09$ \\
ABCS 1 & ABCS 2 & $4.9 \mathrm{e}-01$ & $1.9 \mathrm{e}-01$ & $5.7 \mathrm{e}-01$ & $3.9 \mathrm{e}-01$ & $2.5 \mathrm{e}-01$ & $5.0 \mathrm{e}-01$ \\
\hline \hline
\end{tabular}

\section{Conclusion}

Case studies presented have proven the concept of estimating the angular and position offsets that exist between calibration systems. Simulated calibration results showed that it is possible to recover the induced angles solely based on calibration matrices. These results also displayed the ability to detect the significance of the estimates. The successes of the method were extended to actual balance calibrations with results showing similar trends between two systems each with two replicated calibrations.

From these results, future work should be conducted to further prove the concept by inducing intentional rotations in a calibration system. Results of such a test will validate the developed methodology. An additional improvement to the method would be to significance testing of the position offset. By changing $S_{\Delta F}^{2}$ in Equation 15 to include rotational corrections, then the test will only signify changes solely due to changes in positions.

Methodology developed is expected to preform poorly for calibration systems were a fixed coordinate system does not exist, such as manual calibration stands. In these systems, the rotational offset can change depending on the leveling surface and other factors. Additionally, multiple load points are used that each have their own possible offsets. Improvements in the method should be investigated further to incorporate the ability to estimate independent offsets for each of the possible leveling surfaces and load positions.

\section{References}

\footnotetext{
${ }^{1}$ AIAA, "Recommended Practice: Calibration and Use of Internal Strain Gage Balances with Application to Wind Tunnel Testing," Tech. Rep. R-091-2003, AIAA, 2003.

${ }^{2}$ Shabana, A. A., Computational Dynamics, Wiley, 3rd ed., 2010.

${ }^{3}$ Trimmer, L. L. and Clark, E. L., "Transformation of Axes Systems by Matrix Methods and Applications to Wind Tunnel Data Reduction," Tech. Rep. AEDC-TDR-63-224, Arnold Engineering Develoment Center, 1963.

${ }^{4}$ Myers, R. H. and Montgomery, D. C., Response Surface Methodology, John Wiley \& Sons, 2nd ed., 2002.

${ }^{5}$ Parker, P. A., "A Single-Vector Force Calibration Method Featuring the Modern Design of Experiments," 39th AIAA Aerospace Sciences Meeting and Exhibit, No. 2001-170, AIAA, Reno, NV, January 2001.

${ }^{6}$ Booth, D. and King, D., "Automatic Balance Calibration System (ABCS) Upgrades," 44th AIAA Aerospace Sciences Meeting and Exhibit, No. 2006-516, AIAA, Reno, Nevada, January 2006.
} 